\title{
A Comparison Among Microperimetry, Standard Automated Perimetry and Spectral Domain Optical Coherence Tomography in the Evaluation of the Macula in Glaucoma Eyes with Hemifield Defects
}

Tian Tian $\cdot$ Yu Cai $\cdot$ Mei Li $\cdot$ Yuan Fang $\cdot$ Yingzi Pan

Received: September 21, 2021 / Accepted: November 1, 2021 / Published online: November 20, 2021

(C) The Author(s) 2021

\begin{abstract}
Introduction: To evaluate the correlation between macular integrity assessment (MAIA) and standard automated perimetry (SAP) in detecting macular function damage in glaucoma and to explore the relationship between macular structure and functional damage by using spectral domain optical coherence tomography (SD-OCT).

Methods: This was a cross-sectional study. Seventy patients with glaucoma, with hemifield defects verified by Humphrey 24-2 examination, and 60 normal subjects underwent Humphrey 10-2 and MAIA expert 10-2 examinations. Patients with glaucoma with normal hemifields, as detected by SAP, were divided into a normal hemifield group and a visual field (VF) defect group. The difference in the retina and ganglion cell complex (GCC) thicknesses was analyzed between the abnormal and normal hemifields. Results: Among the 70 glaucoma eyes, the results of MAIA and SAP were consistent for 66
\end{abstract}

T. Tian · Y. Cai $(\bowtie) \cdot$ M. Li · Y. Fang $\cdot$ Y. Pan Department of Ophthalmology, Peking University First Hospital, No.8 Xishiku Street, Xicheng District, Beijing, China

e-mail: cai_yuu@hotmail.comT. Tian

e-mail: tiantian_summer612@yeah.netM. Li

e-mail: limei_5566@126.comY. Fang

e-mail: jasmine_10012891@126.com

Y. Pan

e-mail: panyingzi_pkufh@163.com
$(66 / 70,94.3 \%)$. The others showed SAP hemifield defects, while MAIA was normal (4/70, $5.7 \%)$. There was a good correlation of the mean sensitivity between MAIA and SAP $(P<0.001)$. There also was a good correlation between the mean threshold of MAIA and the mean deviation (MD) of SAP $(P=0.008, r=0.507)$. Among the patients with glaucoma with a normal hemifield, MAIA showed abnormal results in 50 eyes $(50 / 66,75.8 \%)$, which was consistent with the changes in the inner retina and GCC thicknesses. Meanwhile, MAIA showed normal results; there were no significant differences between patients with glaucoma and the normal group in the thicknesses of the inner retina and GCC.

Conclusion: MAIA and SAP have good consistency in detecting macular dysfunction. MAIA can also identify abnormal VFs in the macular regions that may not be detected by SAP, which is consistent with the changes in the GCC thicknesses, suggesting that there may be central VF damage in patients with glaucoma that has not been previously identified.

Keywords: Ganglion cell complex; Glaucoma; Macula; Microperimetry; Optical coherence tomography 


\section{Key Summary Points}

\section{Why carry out this study?}

Central VF defects caused by glaucoma can seriously affect visual function and are not easy to find by routine VF examination at an early stage.

Third-generation microperimetry-MAIA can distinguish the decline of sensitivity between age-related and other macular defects.

This study aimed to evaluate the correlation between MAIA and SAP in detecting macular function damage in glaucoma and to explore the relationship between macular structure and functional damage by using SD-OCT.

What was learned from the study?

MAIA and SAP have good consistency in detecting macular dysfunction.

MAIA can also identify abnormal VFs in the macular regions that may not be detected by SAP, which is consistent with the changes in the GCC thicknesses.

It suggested that there may be central VF damage in patients with glaucoma that has not been previously identified.

\section{INTRODUCTION}

Glaucoma is an optic nerve disease involving progressive damage to both the structure and function of the nerve [1] and is considered to mainly affect the peripheral visual field (VF). In recent years, it has been found that even in the early stage of glaucoma, the central VFs of patients will be affected and accompanied by a loss of retinal ganglion cells (RGCs) in the macular region [2-4]. Defects of the central VF will greatly influence driving and reading ability, which can have profound significance.
Therefore, clinicians should carefully evaluate patients with glaucoma for VF defects occurring near the fixation point.

In some patients with glaucoma, conventional perimetry may be unreliable for monitoring glaucomatous field defects because of reduced fixation stability or eccentric fixation in the foveal-involving field defect from glaucoma or retinal comorbidities [5]. In such patients, fundus-controlled perimetry, microperimetry (MP) may offer a more suitable alternative tool for monitoring disease progression. MP is an increasingly utilized form of perimetry that tracks fundal landmarks to rapidly correct for shifts in the gaze, which enables manual centration of the testing grid at the anatomic fovea. This allows accurate assessment of the central $\mathrm{VF}$, even in the absence of stable and foveal fixation. In recent years, MP has been used to assess macular function in age-related macular degeneration (AMD), macular dystrophy, diabetic retinopathy, and other retinal diseases [6-8]. However, there have been only a few nonage-corrected patients with glaucoma $[9,10]$. Macular integrity assessment (MAIA), a thirdgeneration MP, can distinguish the decline in sensitivity between age-related and other macular defects through the use of an age-corrected normal-vision database, which is absent in firstand second-generation methods.

To explore whether MP can identify central defects early in patients with glaucoma, in this study, we used MAIA to detect visual dysfunction in the normal hemifields of patients with glaucoma, with hemifield damage detected by SAP, as VF defects are more likely to be detected in the normal hemifields of patients who have already suffered glaucomatous hemifield damage. To analyze the relationships between macula functional and structural defects, an RTVue OCT scanner was also used to measure retinal and ganglion cell complex (GCC) thickness changes in the macular region.

\section{METHODS}

Patients with glaucoma with hemifield defects in one eye detected by the Humphrey Swedish Interactive Threshold Algorithm (SITA) 24-2 test 
as well as age- and sex-matched normal subjects were collected from the Department of Ophthalmology at Peking University First Hospital. All subjects signed informed consent forms before inclusion. This study was approved by the Ethics Committee of Peking University First Hospital in accordance with the Declaration of Helsinki.

The inclusion criteria of the glaucoma group were as follows: (1) best corrected visual acuity $($ BCVA) $\geq 0.5$; (2) diopter: $-6.00 \mathrm{~s}<$ spherical diopter $<+4.00 \mathrm{~s}, \quad-3.00 \mathrm{c}<$ cylindrical diopter $<+3.00 \mathrm{c}$, anisometropia $<2.0 \mathrm{D}$; (3) glaucomatous optic neuropathy, defined as a cup-to-disc ratio greater than 0.6 , asymmetry of the cup-to-disc ratio $\geq 0.2$ between eyes, and the presence of local or diffuse retinal nerve fiber layer (RNFL) defects or neuroretinal rim defects in the absence of any other abnormalities that could explain such findings; (4) a glaucomatous VF, defined as glaucoma hemifield test (GHT) results outside normal limits on at least two consecutive baseline tests and the presence of at least three contiguous test points within the same hemifield on the pattern deviation plot at $P<1 \%$, with at least one point $<$ $0.5 \%$, excluding points on the edge of the field or those directly above and below the blind spot; (5) a VF defect involving the central 16 test points (at least one point with $P<1 \%$ ) according to the standard automated perimetry (SAP) 24-2 test [11]; and (6) either a superior or inferior hemifield VF defect according to the SAP 24-2 test.

The exclusion criteria of the glaucoma group were as follows: (1) VF defects in both the superior and inferior hemifields according to the SAP 24-2 test; (2) macular diseases such as age-related macular disease and macular holes; (3) retinal and optic nerve diseases, uveitis, eye trauma, and internal eye surgery history; and (4) diabetes, hypertension, and other systemic diseases.

The inclusion criteria of the normal subjects were as follows: (1) $\mathrm{BCVA} \geq 0.8$; (2) diopter: - $6.00 \mathrm{~s}<$ spherical mirror $<+4.00 \mathrm{~s}$, - $3.00 \mathrm{c}<$ cylindrical diopter $<+3.00 \mathrm{c}$, anisometropia $<2 \mathrm{~s}$; (3) intraocular pressure $(\mathrm{IOP}) \leq 21 \mathrm{mmHg}$; (4) GHT results within normal limits; and (5) no glaucomatous optic nerve defects.

The exclusion criteria of the normal subjects were macular diseases, diabetes, hypertension, and other systemic diseases.

All patients received a routine ophthalmic examination, including a visual acuity test with refraction, slit lamp examination, fundus examination, IOP, gonioscopy, Humphrey perimetry, MAIA, and optical coherence tomography (OCT).

The Humphrey perimetry (Humphrey Field Analyzer model 750i, Carl Zeiss Meditec, Dublin, CA, USA) SITA 24-2 and 10-2 procedures were chosen. VF test results that met the following conditions were considered reliable: (1) fixation loss rate $<20 \%$; (2) false positive rate $<15 \%$; and (3) false negative rate $<15 \%$. Each subject had at least two reliable VF test results, the second of which was used in this study.

For all subjects, the MAIA (CenterVue, Padova, Italy) expert 10-2 algorithm was used to measure retinal sensitivity. The procedure was performed in a dim room without any pupil dilation. The following parameters were used in the current study: a 68-stimuli grid covering the central $10^{\circ}$ of the retina, strategy stimulus size Goldmann III, background luminance set to 4 apostilb (asb), maximum luminance of $1000 \mathrm{asb}$, and a stimulus dynamic range of $36 \mathrm{~dB}$.

An Optovue RTVue-100 (software version 6.1; Optovue, Fremont, CA) spectral domain (SD)-OCT was used for the examinations. The scanning wavelength of the device was $840 \pm 10 \mathrm{~nm}$. The examinations were performed without mydriasis. Internal fixation was used, and the ethnicity was selected as "Chinese". Two scanning modes were used for each subject: "MM6/radial slicer" and "GCC mode". Completely scanned images with a signal strength indicator $\geq 35$ were selected for storage.

\section{Statistical Analysis}

One eye was randomly selected by a random number table for the normal subjects, and the 
eye with the hemifield defect was selected for the glaucoma group. If both eyes met the inclusion criteria in the glaucoma group, the eye with the smaller mean deviation (MD) value was selected. Enumeration data were analyzed by the chi-square test. Measurement data are described by the mean \pm standard deviation (SD), and the differences between two groups and among three groups were analyzed by the $t$ test and one-way analysis of variance (ANOVA), respectively. A mixed-effect model and Pearson correlation analysis were used to calculate the correlation between the two variables. The consistency between the MAIA average threshold and the Humphrey 10-2 MD was compared by means of the kappa statistic. Statistical software SPSS 16.0 (SPSS, Inc., Chicago, USA) was used to perform these analyses, and $P<0.05$ was considered statistically significant.

\section{RESULTS}

1. Seventy patients with glaucoma with hemifield defects and 60 age- and sex-matched normal control patients were included (Table 1).

2. Correlation of average light sensitivity between MAIA and SAP

The mixed-effect model showed that there was a moderate positive correlation of the average light sensitivity between MAIA and SAP $(P<0.001)$ (Table 2$)$.
Table 2 Correlation of average light sensitivity between MAIA and SAP

\begin{tabular}{lllrr}
\hline & Coefficient & $\begin{array}{l}\text { Standard } \\
\text { error }\end{array}$ & \multicolumn{1}{l}{$\boldsymbol{Z}$} & \multicolumn{1}{l}{$\boldsymbol{P}$} \\
\hline MAIA & 0.403 & 0.185 & 21.80 & $<0.001$ \\
Constant & 2.322 & 1.148 & 2.02 & 0.043 \\
\hline
\end{tabular}

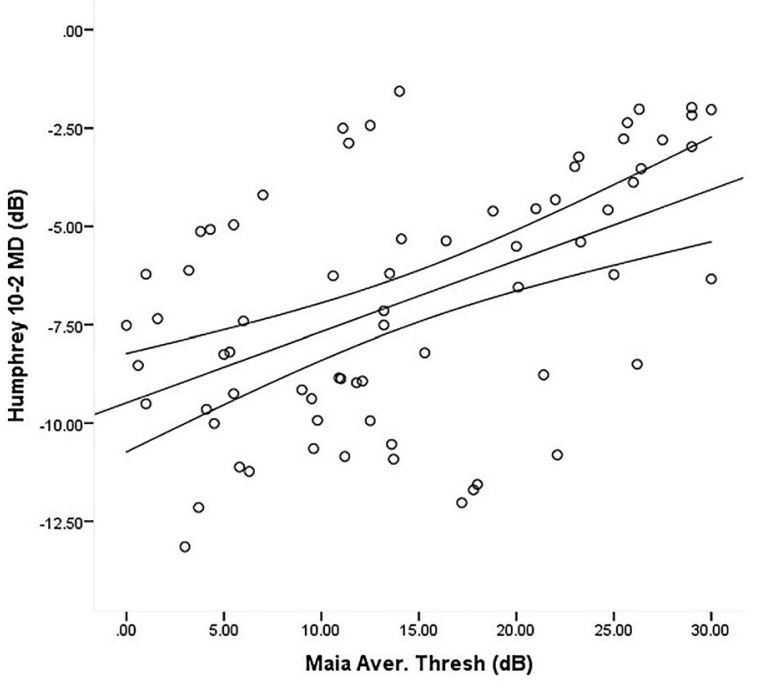

Fig. 1 Moderate positive correlation between the MAIA average threshold and Humphrey 10-2 MD $(P=0.008$, $r=0.507$ )

Table 1 General characteristics of patients with glaucoma and normal subjects

\begin{tabular}{lllllll}
\hline & $\boldsymbol{n}$ & Age (years) & Sex $(\mathbf{M} / \mathbf{F})$ & BCVA $(\log M A R)$ & MD (dB) & PSD (dB) \\
\hline Normal subjects & 60 & $54.0 \pm 14.7$ & $28 / 32$ & 0 & $-1.33 \pm 1.01$ & $1.01 \pm 0.11$ \\
Patients with glaucoma & 70 & $54.8 \pm 17.8$ & $32 / 38$ & 0.097 & $-7.23 \pm 5.51$ & $8.61 \pm 5.24$ \\
$P$ & & 0.259 & $0.939^{\mathrm{a}}$ & $0.032^{\mathrm{b}}$ & $0.000^{* *}$ & $0.000^{* *}$ \\
Subtype & & & & & & \\
NTG & 44 & & & & $-7.48 \pm 5.46$ & $9.30 \pm 5.21$ \\
POAG & 26 & & & & $-8.62 \pm 6.90$ & $9.28 \pm 5.90$ \\
$P$ & & & & & 0.064 & 0.071 \\
\hline
\end{tabular}

${ }^{\mathrm{a}}$ Chi-square test; ${ }^{\mathrm{b}}$ Mann-Whitney test

${ }^{* *} P<0.01$ 


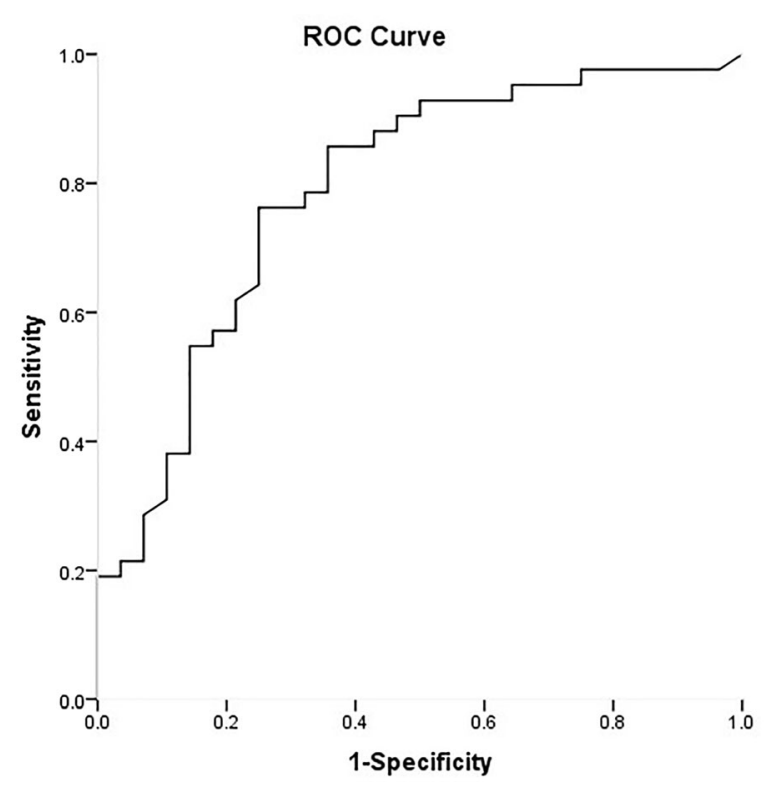

Fig. 2 Receiver operating characteristic curve for the MAIA average threshold and the Humphrey 10-2 MD in patients with glaucoma

3. Correlation between the MAIA average threshold and the Humphrey 10-2 MD

There was a moderate positive correlation between the MAIA average threshold and the Humphrey 10-2 MD among patients with glaucoma $(P=0.008, r=0.507)$, as shown in Fig. 1.

4. According to the MD, early stage $(\mathrm{MD} \geq-6 \mathrm{~dB})$ glaucoma was defined as 0 (28 eyes), and middle $(-6 \mathrm{~dB}>\mathrm{MD} \geq-12 \mathrm{~dB})$ and late stage $(\mathrm{MD}<-12 \mathrm{~dB})$ glaucoma was defined as 1 (42 eyes). A receiver operating characteristic (ROC) curve was generated (Fig. 2), and the Youden index was calculated. The consistency between the MAIA average threshold and the Humphrey 10-2 MD was compared by means of the kappa statistic. The sensitivity was 0.762 , the specificity was 0.750 , the Youden index was 0.512, and the kappa statistic was 0.503 .

5. Analysis of VF and hemifield thicknesses of the inner retina and GCC

Both the Humphrey 10-2 program and MAIA showed hemifield defects in 66 eyes $(66 / 70,94.3 \%)$. The others showed Humphrey hemifield defects, while MAIA was normal $(4 / 70,5.7 \%)$. In 50 eyes, the other hemifield was abnormal according to MAIA (50/66, 75.8\%), while the Humphrey 10-2 program indicated that it was normal. We defined these eyes as group I (Fig. 3). Both MAIA and Humphrey examination showed that the hemifields of the remaining 16 eyes $(16 / 66,24.2 \%)$ were normal (the results of the two examinations were consistent); these eyes were defined as group II (Fig. 4). The hemifield macular inner retina and GCC thicknesses in group I were significantly thinner than those in the normal control group $(P<0.05)$. There was no significant difference in the macular inner retina and GCC thicknesses between group II and the normal group $(P>0.05)$ (Table 3).

\section{DISCUSSION}

In this study, the mixed-effect model showed that there was a positive correlation of the average light sensitivity between MAIA and SAP $(P<0.001)$. We also found a moderate positive correlation between the MAIA average threshold and Humphrey 10-2 MD $(P=0.008$, $r=0.507)$. The consistency between the MAIA average threshold and the Humphrey 10-2 MD was compared by means of the kappa statistic. The sensitivity was 0.762 , the specificity was 0.750 , the Youden index was 0.512, and the kappa statistic was 0.503. Both MAIA and SAP had good consistency in detecting central VF defects in patients with glaucoma. In previous studies, Orzalesi et al. found a good correlation between microperimetry and SLO (scanning laser ophthalmoscopy) using a $40^{\circ}$ field of view and SAP of the central $30^{\circ}[12,13]$. Comparative studies on MP and SAP have shown that there is a good quantitative correlation of numerical data between the two perimeters in advanced cases of glaucoma $[11,14-16]$. In our study, the MAIA expert 10-2 program used in the examination consisted of the central $10^{\circ}$ and 56 detection points, the same as the Humphrey 10-2 program. The results showed that there was a good correspondence between MAIA and 

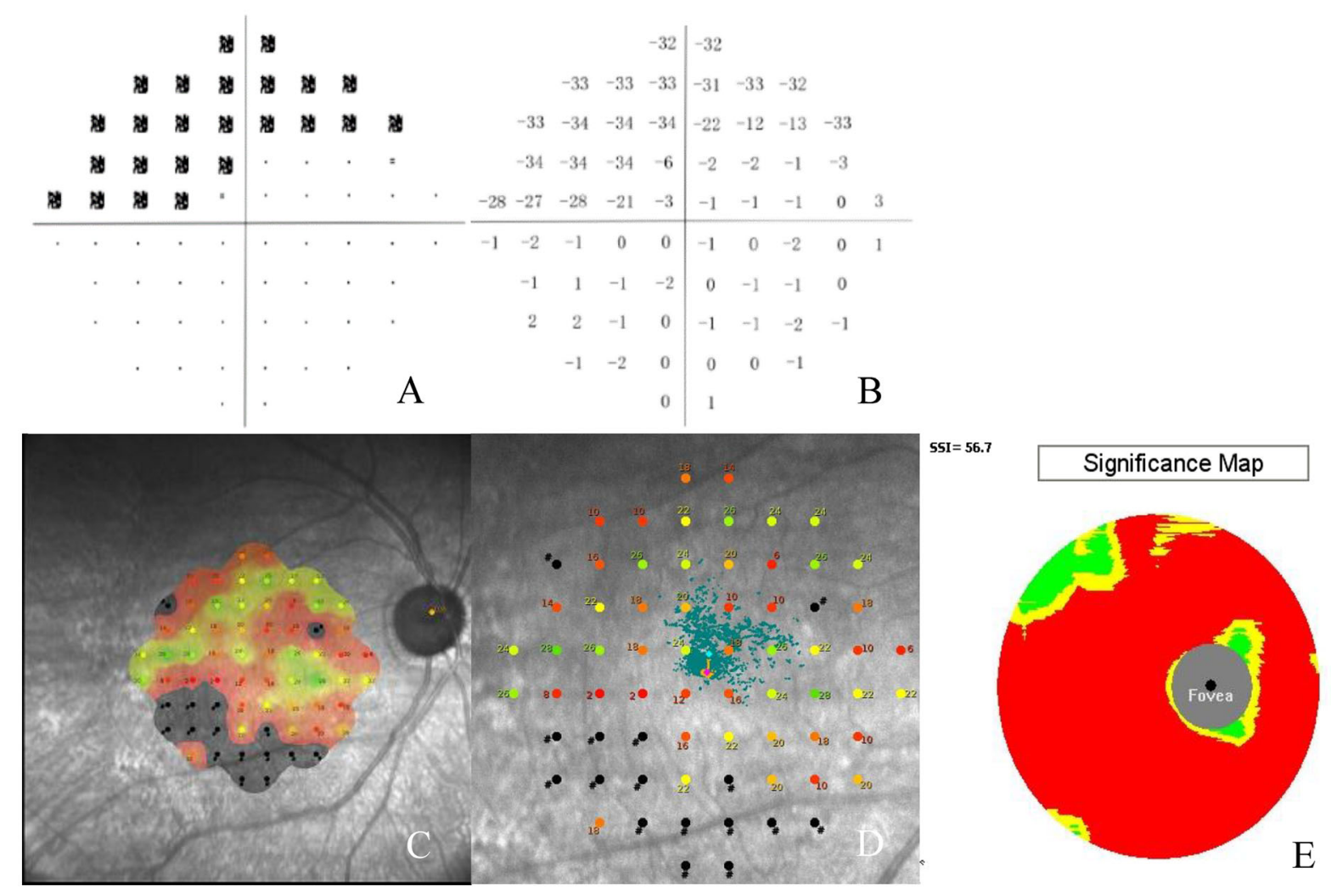

Fig. 3 Group I: Inconsistent results between the Humphrey and MAIA examinations. Humphrey 10-2 showed a VF defect in the superior hemifield and no obvious abnormality in the inferior hemifield $(\mathbf{a}, \mathbf{b})$. MAIA showed both superior and inferior VF defects (c, d) (red represents

SAP. Additionally, the light sensitivity of MAIA and SAP was compared, which may form the basis for future of macular function in glaucoma.

According to the results, when SAP was normal but MAIA detected defects, the thicknesses of the corresponding inner retina and GCC in group I were thinner than those in the normal group $(P<0.05)$. At the same time, there were no significant differences in the inner retina and GCC thicknesses between group II and normal subjects $(P>0.05)$ when VF defects were not detected by either SAP or MAIA. The results of our study showed that MAIA could detect more subtle glaucomatous functional damage $(50 / 66,75.8 \%)$ than SAP, which is consistent with the thinning of the GCC detected by SD-OCT. Recent studies have suggested that macular OCT imaging could be abnormal, yellow represents suspect, and green represents normal), and OCT showed that the whole GCC was thinner in patients with glaucoma than in normal subjects (e)

helpful for the detection of disease deterioration [17]. It is generally accepted that measuring both structure and function could lead to more efficient and timely detection of glaucoma progression to optimize the management of patients with glaucoma [18-20]. Lima et al. found that $21 \%$ of the normal SAP quadrants had an abnormal corresponding MP result; a corresponding significant reduction in the total macular thickness measured by OCT was present in $75 \%$ of these quadrants $[11,13]$. This was consistent with the results of our study. We also measured the thickness of the GCC in the macular area, and the results suggest that there may be more patients with minor paracentric dysfunction that could not be detected by SAP.

Recently, Phuljhele et al. also found that patients with early glaucoma with a nasal step on SAP had significant absolute or moderate 


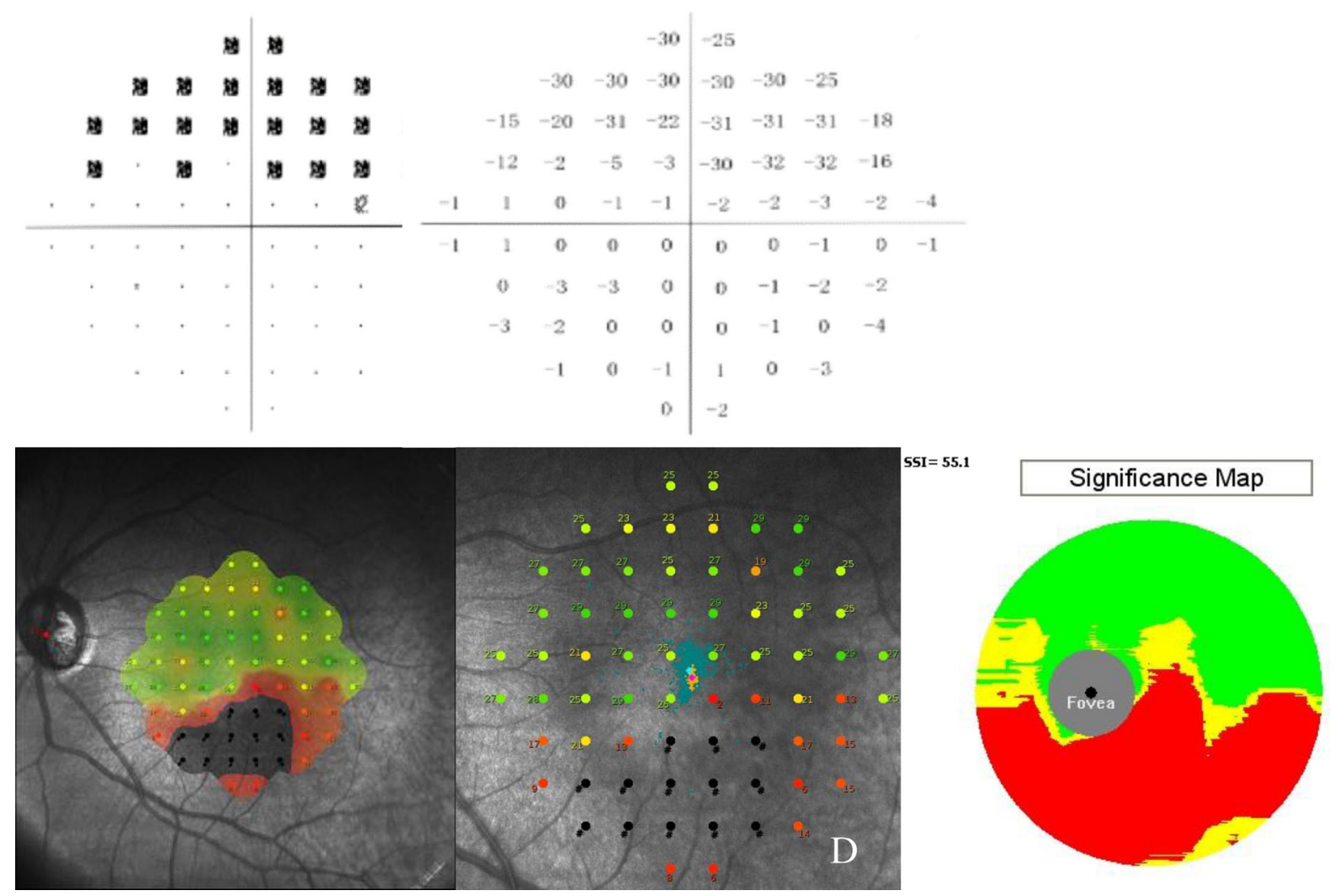

Fig. 4 Group II: Consistent results between the Humphrey and MAIA examinations. Humphrey 10-2 showed a VF defect in the superior hemifield and no obvious abnormalities in the inferior hemifield (a, b). MAIA

microperimetric defects in the corresponding temporal retinal quadrants, $8-10^{\circ}$ off fixation. An additional mild defect was present in $10-52 \%$ of eyes, extending till $4^{\circ}$ from fixation and involving almost the entire hemisphere [21]. Therefore, MP identified abnormal retinal sensitivities over a much wider area in eyes as compared with SAP. Furthermore, the combination of macular structural and functional examination is of great importance for glaucoma diagnosis and disease progression monitoring [10]. Kita et al. found that circumpapillary (cp) MP sensitivity and the cp RNFL thickness showed a similar diagnostic power [22]. Tham et al. showed that visual sensitivity measurements of both SAP and MP demonstrated a similar relationship with ganglion cell-inner plexiform layer measurements of SD-OCT at the macula in glaucoma [23]. Therefore, MP can be a simple and more reliable showed a consistent inferior VF defect corresponding to the Humphrey 10-2 defect (c, d). OCT also showed that only the inferior half of the GCC was thinner in patients with glaucoma than in normal subjects (e)

method to determine the retinal sensitivity at specific loci and can also detect early progression, which is otherwise not observed on SAP.

In previous studies, SD-OCT was capable of detecting the difference in GCC thickness between patients with early primary open angle glaucoma (POAG) and normal controls [24], and approximately $2 / 3$ of patients with advanced POAG had thinner GCC [25]. Wang found that the thickness of the RNFL and $\mathrm{RGC}^{+}$ layers (RGC + IPL) in the macular areas of patients with glaucoma was thinner than that of the normal control group, while the thicknesses of the inner nuclear layer and photoreceptor cell layer were basically similar between the two groups [26]. A large number of studies have shown that in the diagnosis of glaucoma, the GCC thickness is more effective than the macular thickness, while there is no significant difference between the GCC and RNFL. SD-OCT 
Table 3 Comparisons of the macular inner retina and GCC thicknesses between group I and II with normal subjects

\begin{tabular}{llllllll}
\hline & Group I & Group II & $\begin{array}{l}\text { Normal } \\
\text { subjects }\end{array}$ & $\boldsymbol{P}$ & $\boldsymbol{P}_{\text {I-Normal }}$ & $\boldsymbol{P}_{\text {II-Normal }}$ & $\boldsymbol{P}_{\text {I-II }}$ \\
\hline$n$ & 50 & 16 & 60 & & & & \\
$\begin{array}{c}\text { Parafovea thickness, } \\
\text { hemisphere }(\mu \mathrm{m})\end{array}$ & $103.76 \pm 13.70$ & $113.63 \pm 16.76$ & $121.80 \pm 12.00$ & $0.000^{* *}$ & $0.000^{* *}$ & 0.128 & 0.063 \\
$\begin{array}{c}\text { Parafovea thickness, } \\
\text { hemisphere }(\mu \mathrm{m})\end{array}$ & $105.28 \pm 12.11$ & $114.50 \pm 16.93$ & $124.70 \pm 11.40$ & $0.000^{* *}$ & $0.000^{* *}$ & 0.068 & 0.073 \\
$\begin{array}{c}\text { GCC thickness, } \\
\text { hemisphere }(\mu \mathrm{m})\end{array}$ & $79.65 \pm 8.13$ & $91.60 \pm 8.38$ & $95.38 \pm 6.33$ & $0.000^{* *}$ & $0.000^{* *}$ & 0.200 & $0.000^{* *}$ \\
$\begin{array}{c}\text { Perifovea thickness, } \\
\text { hemisphere }(\mu \mathrm{m})\end{array}$ & $88.76 \pm 11.50$ & $95.75 \pm 13.20$ & $101.83 \pm 8.02$ & $0.000^{* *}$ & $0.000^{* *}$ & 0.140 & 0.097 \\
$\begin{array}{c}\text { Perifovea thickness, } \\
\text { hemisphere }(\mathrm{m})\end{array}$ & $87.44 \pm 8.89$ & $95.63 \pm 10.49$ & $100.87 \pm 7.21$ & $0.000^{* *}$ & $0.000^{* *}$ & 0.120 & $0.019^{*}$ \\
\hline
\end{tabular}

One-way ANOVA was used to compare the three groups

${ }^{*} P<0.05,{ }^{* *} P<0.01$

was used to measure the GCC thickness in the macular area in our study, and compared with other modalities, it can better reflect the structural changes in the macular area in glaucoma.

Furthermore, the combination of macular structural and functional examination is of great importance for glaucoma diagnosis and disease progression monitoring [10]. Sato et al. found that there was a structural and functional relationship between the retinal thickness measured by OCT and the central VF sensitivity measured by MP in patients with glaucoma. However, the macular parameter used in that study was the thickness of the retinal ganglion cell layer and the inner plexiform layer (GCA), which did not contain the RNFL [27].

With regard to potential limitations, the number of subjects in this study was relatively small; however, a further study with a larger number of subjects should be able to address this issue. Many of our patients had normal tension glaucoma (NTG), and they may present more often with central scotomas than patients with hypertension glaucoma, suggesting that our work should be replicated for other forms of glaucoma.

\section{CONCLUSIONS}

The mean macular sensitivity measured by MAIA has a good correlation with SAP among patients with glaucoma with hemifield defects. MAIA can detect the abnormal functioning of the macula in patients with glaucoma with a normal VF according to SAP but an already defective GCC according to OCT. This suggests that there may be minor paracentric dysfunctions that have not been found in glaucoma before.

\section{ACKNOWLEDGEMENTS}

Funding. This research's data collection, and the journal's Rapid Service Fee, was funded by Natural Science Foundation of Beijing Municipal (Grant No. 7202208) and the Youth Clinical Research Project of Peking University First Hospital (No. 2019CR01).

Authorship. All named authors meet the International Committee of Medical Journal Editors (ICMJE) criteria for authorship for this article, take responsibility for the integrity of 
the work as a whole, and have given their approval for this version to be published.

Author Contributions. Tian Tian collected the data and drafted the manuscript. Yu Cai designed the study, performed the surgeries, and was involved in collecting the data and drafting the manuscript, and also critically revised the manuscript and has given final approval of the version to be published. Mei Li participated in the design of the study and the collection of the data. Yuan Fang and Yingzi Pan participated in the design of the study. All authors read and approved the final manuscript.

Disclosures. Tian Tian, Yu Cai, Mei Li, Yuan Fang, Yingzi Pan have nothing to declare. Neither honoraria nor payments were made for authorship of this article. All authors declare no proprietary interest.

Compliance with Ethics Guidelines. All procedures performed in studies involving human participants were in accordance with the ethical standards of the institutional and/or national research committee and with the 1964 Helsinki declaration and its later amendments or comparable ethical standards.

Data Availability. The datasets generated during and/or analyzed during the current study are available from the corresponding author on reasonable request.

Informed Consent. The study protocol was approved by Peking University First Hospital (Protocol. No.201916).

Open Access. This article is licensed under a Creative Commons Attribution-NonCommercial 4.0 International License, which permits any non-commercial use, sharing, adaptation, distribution and reproduction in any medium or format, as long as you give appropriate credit to the original author(s) and the source, provide a link to the Creative Commons licence, and indicate if changes were made. The images or other third party material in this article are included in the article's Creative Commons licence, unless indicated otherwise in a credit line to the material. If material is not included in the article's Creative Commons licence and your intended use is not permitted by statutory regulation or exceeds the permitted use, you will need to obtain permission directly from the copyright holder.

Open Access. This article is licensed under a Creative Commons Attribution-NonCommercial 4.0 International License, which permits any non-commercial use, sharing, adaptation, distribution and reproduction in any medium or format, as long as you give appropriate credit to the original author(s) and the source, provide a link to the Creative Commons licence, and indicate if changes were made. The images or other third party material in this article are included in the article's Creative Commons licence, unless indicated otherwise in a credit line to the material. If material is not included in the article's Creative Commons licence and your intended use is not permitted by statutory regulation or exceeds the permitted use, you will need to obtain permission directly from the copyright holder. To view a copy of this licence, visit http://creativecommons.org/licenses/by$\mathrm{nc} / 4.0 /$.

\section{REFERENCES}

1. Weinreb NR, Aung T, Medeiros FA. The pathophysiology and treatment of glaucoma. JAMA. 2014;311(18):1901-11.

2. Hirooka K, Misaki K, Nitta E, Ukegawa K, Sato S, Tsujikawa A. Comparison of Macular Integrity Assessment $\left(\mathrm{MAIA}^{\mathrm{TM}}\right), \mathrm{MP}-3$, and the Humphrey field analyzer in the evaluation of the relationship between the structure and function of the macula. PLoS One. 2016;11(3):e0151000.

3. Fujita K, Yasuda N, Oda K, Yuzawa M. Reading performance in patients with central visual field disturbance due to glaucoma. Nippon Ganka Gakkai Zasshi. 2006;110(11):914-8.

4. Coeckelbergh TRM. The effect of visual field defects on driving performance: a driving simulator study. Arch Ophthalmol. 2002;120(11):1509. 
5. Joseph G, Jeffrey G. Prevalence of comorbid retinal disease in patients with glaucoma at an academic medical center. Clin Ophthalmol. 2015. https://doi. org/10.2147/OPTH.S85851.

6. Ooto S, Suzuki M, Vongkulsiri S, Sato T, Spaide RFJR. Multimodal visual function testing in eyes with nonexudative age-related macular degeneration. Retina. 2015;35(9):1726.

7. Cennamo G, Vecchio EC, Finelli M, Velotti N, De Crecchio G. Evaluation of ischemic diabetic maculopathy with Fourier-domain optical coherence tomography and microperimetry. Can J Ophthalmol. 2015. https://doi.org/10.1016/j.jcjo.2014.08. 005 .

8. Marco DV, Carlo L, Marco N, Grignolo FM, Fea AM. Microperimetric assessment after epiretinal membrane surgery: 4-year follow-up. J Ophthalmol. 2016;2016:1-5.

9. Kita Y, Holló G, Murai A, Kita R, Saito T, Hirakata AJCO. Circumpapillary structure-function relationships with microperimetry and spectral domain optical coherence tomography in glaucoma: a pilot study. Clin Ophthalmol. 2018;12:2535-44.

10. Rao HL, Hussain RSM, Januwada M, et al. Structural and functional assessment of macula to diagnose glaucoma. Eye (Lond). 2017;31(4):593-600.

11. Lima VC, Prata TS, De Moraes CGV, et al. A comparison between microperimetry and standard achromatic perimetry of the central visual field in eyes with glaucomatous paracentral visual-field defects. Br J Ophthalmol. 2010;94(1):64-7.

12. Arrico L, Giannotti R, Fratipietro M, Malagola R. Fascicular visual field defects in open-angle glaucoma: evaluation with microperimetry. J Ophthalmol. 2016;2016:8274954. https://doi.org/10.1155/ $2016 / 8274954$

13. Orzalesi N, Miglior S, Lonati C, Rosetti LJVR. Microperimetry of localized retinal nerve fiber layer defects. Vis Res. 1998;38(5):763-71.

14. Okada K, Watanabe W, Koike I, Tsumamoto Y, Mishima HK. Alternative method of evaluating visual field deterioration in very advanced glaucomatous eye by microperimetry. Jpn J Ophthalmol. 2003;47(2):178-81.

15. Rensch F, Jonas JB. Direct microperimetry of alpha zone and beta zone parapapillary atrophy. $\mathrm{Br} \mathrm{J}$ Ophthalmol. 2008;92(12):1617.

16. Öztürk F, Yavas GF, Küsbeci T, Ermis SS. A comparison among Humphrey field analyzer, microperimetry, and Heidelberg retina tomograph in the evaluation of macula in primary open angle glaucoma. J Glaucoma. 2008;17(2):118-21.

17. Nickells RW. The cell and molecular biology of glaucoma: mechanisms of retinal ganglion cell death. Invest Ophthalmol Vis Sci. 2012;53(5):2476.

18. Lavinsky F, Wu M, Schuman JS, et al. Can macula and optic nerve head parameters detect glaucoma progression in eyes with advanced circumpapillary retinal nerve fiber layer damage? Ophthalmology. 2018;125(12):1907-12.

19. Hou WH, Lin C, Leung CKS. Integrating macular ganglion cell inner plexiform layer and parapapillary retinal nerve fiber layer measurements to detect glaucoma progression. Ophthalmology. 2018;125(6):822-31.

20. Shin JW, Sung KR, Park SWJO. Patterns of progressive ganglion cell-inner plexiform layer thinning in glaucoma detected by OCT. Ophthalmology. 2018;125(10):1515-25.

21. Phuljhele S, Angmo D, Aalok L, Parwal S, Sihota R. Functional evaluation of the macular area in early glaucoma using microperimetry. Indian J Ophthalmol. 2021;69(4):876-81.

22. Kita Y, Soutome N, Horie D, Kita R, Holló G. Circumpapillary ganglion cell complex thickness to diagnose glaucoma: a pilot study. Indian J Ophthalmol. 2017;65(1):41.

23. Tham YC, Li X, Wong TY, Quigley HA, Cheng CY. Global prevalence of glaucoma and projections of glaucoma burden through 2040: a systematic review and meta-analysis. Ophthalmology. 2014;121(11):2081-90.

24. Fan N, Huang LN, He J, Shen XL, Kun Z, Hong-bo C. Quantitative measurement of macular ganglion cell complex thickness by spectral domain optical coherence tomography. Chin J Exp Ophthalmol. 2012;30(8):743-7.

25. Tan O, Chopra V, Lu TH, Schuman JS, Huang DJO. Detection of macular ganglion cell loss in glaucoma by Fourier-domain optical coherence tomography. Ophthalmology. 2009;116(12):2305-2314.e2302.

26. Wang M. Measurement of local retinal ganglion cell layer thickness in patients with glaucoma using frequency-domain optical coherence tomography. Arch Ophthalmol. 2009;127(7):875.

27. Sato S, Hirooka K, Baba T, et al. Correlation between the ganglion cell-inner plexiform layer thickness measured with cirrus HD-OCT and macular visual field sensitivity measured with microperimetry. Invest Ophthalmol Vis Sci. 2013;54(4):3046. 